\title{
Circunferência escrotal e características do sêmen de touros Nelore aos 18 meses de idade
}

\section{Scrotal circumference and semen quality of 18 month Nelore bulls}

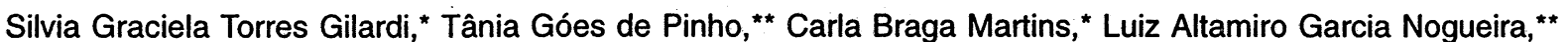 \\ Paulo Augusto Pinto, ${ }^{\star \star \star}$ Marcelo Esteves Caldas, ${ }^{\star \star \star \star}$ Magno Roberto de Souza. ${ }^{\star \star \star \star \star}$
}

\begin{abstract}
Resumo
Circunferência escrotal (CE) e qualidade do sêmen (motilidade e patologia) de 100 touros Nelore aos 18 meses de idade foram estudados objetivando obter subsídios que auxiliem a seleção de touros jovens Nelore para a reprodução. A média de circunferência escrotal foi de $24,8 \pm 2,7 \mathrm{~cm}$, sendo que $54 \%$ apresentaram CE abaixo de $27 \mathrm{~cm}$. Trinta e quatro por cento dos touros apresentaram sêmen oligospérmico ou azoospérmico. A grande maioria $(94,8 \%)$ dos animais com CE < $23 \mathrm{~cm}$ foi oligo/azoospérmico. Diferença significativa $(p<0,05)$ foi encontrada entre as médias de CE de touros com sêmen denso e oligospérmico e $(p<0,05)$ entre as médias de CE de oligospérmicos e azoospérmicos. O percentual médio da motilidade progressiva e patologia do sêmen de touros Nelore aos 18 meses de idade foi de $51,4 \pm 7,2 \%$ e $65,6 \pm 5,4 \%$ respectivamente. Houve diferença significativa entre as médias de motilidade progressiva $(p<0,05)$ e de patologia espermática $(p<0,05)$ dos animais com CE $\geq 23<27 \mathrm{~cm}$ e $\geq 27 \mathrm{~cm}$. A correlação entre CE e motilidade progressiva do sêmen e entre CE e patologia espermática foi de $r=0,34$ e $r=-0,46$ respectivamente. Conclui-se que a maioria dos touros Nelore aos 18 meses de idade são púberes porém a qualidade seminal ainda é baixa. A CE pode ser considerada eficaz na seleção de touros jovens por apresentar nesta ocasião correlação com parâmetros estudados de qualidade seminal.
\end{abstract}

Palavras-chave: touros Nelore; circunferência escrotal; sêmen.

\begin{abstract}
Scrotal circumference (SC) and semen quality (sperm motility and morphologic abnormalities) of 18 month Nelore bulls were studied, in order to gather information that will help the selection of Nelore bull calves for reproduction purposes. Average scrotal circumference was $24.8 \pm 2,7 \mathrm{~cm}$; of which $54 \%$ presented SC below $27.0 \mathrm{~cm}$. Thirty-four percent of the bulls presented oligospermia or azoospermia. Most of the animals with SC below $23.0 \mathrm{~cm}(94.8 \%)$ presented oligo/azoospermia. A significant difference $(p<0.05)$ was found between the SC averages of bulls with dense and oligospermic semen; as well as $(p<0.05)$ between the SC averages of bulls with oligospermia and azoospermia. The average percentage of progressive motility and morphologic abnormalities on the semen of 18 month Nelore bulls was $51.4 \pm 7.2 \%$ and $65.6 \pm 5.4 \%$, respectively. There was a significant difference between the averages of sperm motility $(p<0.05)$ and morphologic abnormalities of animals with SC $323<27$ e $327 \mathrm{~cm}$. The correlations between SC and sperm motility and between SC and morphologic abnormalities were $r=0.34$ and $r=-0.46$, respectively. It can be concluded that most 18 month Nelore bulls are in puberty, however the quality of semen is still poor. The SC can be considered an efficient method of bull calves selection for it revealed a correlation with the semen quality parameters studied herein.
\end{abstract}

Keywords: Nelore bulls; scrotal circunference; semen.

\section{Introdução}

$\mathrm{Na}$ seleção de touros de raças de corte para a reprodução buscam-se características que possam precocemente estimar o desenvolvimento ponderal e o potencial reprodutivo desses animais. Uma das caracteristicas mais importantes nos programas de seleção de touros para o melhoramento da raça Nelore é, na atualidade, a circunferência escrotal. Trata-se de uma característica que apresenta facilidade de avaliação, correlação positiva com idade, características ponderais e o peso dos testículos (Pinto et al., 1989; Cardoso, 1977), sendo que este tem alta correlação com produção espermática diária e alta qualidade do sêmen (Toelle, 1985; Rao Veeramachaneni et al., 1986). Sabe-se que

*Grad. UFF.

**Docente Dr. Fisiop. Reprod. UFF - Rua Moreira César, 129/304 - Icaraí - Niterói,RJ - CEP 24230-050.

***Pós-grad. UFF.

****Docente Dr. USP

*****Med. Vet. Manah. 
espermatozóides maturos já podem ser encontrados nos túbulos seminíferos de touros Nelore aos 15 meses de idade (Cardoso e Godinho, 1979). Porém, um touro jovem é considerado púbere quando seu sêmen tem pelo menos $50 \times 10^{6} \mathrm{sptz} /$ $\mathrm{ml}$ e $10 \%$ de motilidade progressiva. Após atingida a puberdade, há ainda um gradual aumento do tamanho dos testículos, volume do ejaculado e produção total de sptz (Foote et al., 1976). $\mathrm{Na}$ avaliação do sêmen, a motilidade e a morfologia espermática são as medidas mais utilizadas para predizer a qualidade seminal, podendo revelar distúrbios bioquímicos do sêmen e alterações da espermatogênese (Fonseca, 1995). Sabe-se que existe alta correlação entre peso e puberdade dos animais, porém, segundo Valle Filho et al. (1997), a correlação entre idade cronológica e sexual pode ser tão importante quanto a do peso corporal, dependendo do parâmetro considerado. O objetivo deste trabalho foi obter informações sobre circunferência escrotal, qualidade seminal do primeiro ejaculado de touros Nelore aos 18 meses de idade, buscando correlações entre os parâmetros e subsídios para a seleção de reprodutores mais precocemente.

\section{Material e métodos}

Foram estudados 100 touros da raça Nelore (Bos taurus indicus) da linhagem Längruber, com idade de 18 meses $( \pm 1$ mês) criados extensivamente, pertencentes à fazenda Mundo Novo, no município de Brotas, SP. Os animais foram submetidos a anterior exame andrológico, não sendo constatada nenhuma anomalia no sistema genital. A circunferência escrotal foi mensurada em centímetros na região de seu maior diâmetro. O sêmen foi colhido por eletroejaculação e avaliado imediatamente ao microscópio óptico e classificado de forma subjetiva quanto a densidade (denso, oligospérmico, ou azoospérmico) e a motilidade progressiva (\%). Posteriormente foi determinado o percentual de patologia espermática em microscopia de contraste de fase em amostras colhidas e mantidas em solução de formol salina tamponada.

A análise estatística envolveu o cálculo de medidas de tendência central (média), medidas de dispersão (desvio-padrão), comparação entre médias (teste t) e correlação. $O$ modelo estatístico utilizado no estudo das médias de circunferência escrotal foi: $Y i j=m+D E i$ + eij, onde $m$ é a média, o DEi o efeito da densidade na característica estudada e eij o erro aleatório.

\section{Resultados}

Resultados da Tabela 1 mostram que, dos 100 animais avaliados quanto à densidade seminal, $66 \%$ tiveram sêmen clas-

Tabela 1- Número de animais e média de circunferência escrotal (CE) de touros Nelore aos 18 meses de idade, classificados em grupos de acordo com a densidade do sêmen

\begin{tabular}{lcc}
\hline Densidade do Sêmen & n. & Média de CE \\
\hline Denso & 66 & $27,6^{\mathrm{a}}$ \\
Oligospérmico & 10 & $24,3^{\mathrm{b}}$ \\
Azoospérmico & 24 & $22,5^{\mathrm{c}}$ \\
Total $^{-}$ & 100 & $24,8 \pm 2,7$ \\
\hline
\end{tabular}

Letras diferentes $=$ diferença significativa $(p<0,05)$. sificado como denso, $10 \%$ como oligospérmico e $24 \%$ como azoospérmico. A circunferência escrotal média dos 100 animais foi de $24,8 \pm 2,7 \mathrm{~cm}$. A diferença entre as médias de circunferência escrotal foi significativa $(p<0,05)$ nos grupos de animais que apresentaram, ao primeiro ejaculado, sêmen denso $(27,6 \mathrm{~cm})$ e oligospérmico $(24,3 \mathrm{~cm})$ e $(p<0,05)$ entre os grupos oligospérmico e azoospérmico.

De acordo com os resultados da Tabela 2 verifica-se que, dos 100 touros classificados em três grupos quanto à circunferência escrotal, $21 \%$ tiveram CE $<23 \mathrm{~cm}, 33 \%$ CE $\geq 23$ e $<27 \mathrm{~cm}$ e $46 \%>27 \mathrm{~cm}$ e que a maioria dos touros $(94,8 \%)$ com CE $<23 \mathrm{~cm}$ apresentou sêmen oligospérmico ou azoospérmico. Cinqüenta e quatro por cento apresentaram CE abaixo de $27 \mathrm{~cm}$.

As médias de motilidade progressiva e de patologia espermática total do sêmen foram de $51,4 \pm 7,2 \%$ e $65,6 \pm 5,4 \%$ respectivamente. A diferença entre a motilidade progressiva do sêmen dos animais dos grupos CE2 (45\%) e CE3 $(59,5 \%)$ foi significativa $(p<0,05)$. Da mesma forma, a diferença entre as médias de patologia espermática total do sêmen dos animais dos grupos CE2 (20\%) e CE3 (31\%) foi também significativa $(p<0,05)$.

A correlação entre motilidade e CE foi de $r=0,34$; entre $C E$ e patologia, $r=-0,46$ e entre motilidade e patologia, $r=-0,56$.

Tabela 2 - Características seminais de touros Nelore aos 18 meses de idade classificados em grupos de acordo com a circunferência escrotal (CE).

\begin{tabular}{|c|c|c|c|c|c|c|}
\hline \multirow{2}{*}{$\begin{array}{l}\text { Perím. Escrotal } \\
\text { (cm) }\end{array}$} & \multirow[t]{2}{*}{$n$} & \multirow{2}{*}{$\begin{array}{c}\text { Oligosp.- } \\
\text { Azoosp. } \\
n\end{array}$} & \multicolumn{2}{|c|}{$\begin{array}{l}\text { \% Motil. } \\
\text { Progress. }\end{array}$} & \multicolumn{2}{|c|}{$\%$ Patol. Esperm. Total } \\
\hline & & & $n$ & $x$ & $n$ & $x$ \\
\hline CE1 $(<23)$ & 21 & 20 & 1 & 50 & 1 & 84 \\
\hline CE2 $(\geq 23<27)$ & 33 & 11 & 23 & $45,6^{a}$ & 20 & $63,9^{c}$ \\
\hline CE3 $(\geq 27)$ & 46 & 3 & 43 & $59,5^{b}$ & 31 & $47,4^{d}$ \\
\hline Total & 100 & 34 & 66 & $51,7 \pm 7,2$ & 52 & $65,6 \pm 5,4$ \\
\hline
\end{tabular}

Médias seguidas por letras diferentes na mesma característica = diferença significativa $(p<0,05)$

\section{Discussão e conclusões}

Touros zebuínos geralmente entram em atividade reprodutiva mais tardiamente do que touros de raças européias. Aos 18 meses de idade, alguns touros Nelore ainda são impúberes e muitos ainda não atingiram a maturidade sexual (Cardoso,1977; Cardoso e Godinho,1979). A seleção de animais para a reprodução visa atualmente não só a qualidade seminal, mas a precocidade dos reprodutores, daí a importância de estudar características que possam estimar qualidade seminal em grupos de animais que ainda não atingiram a maturidade sexual, predizendo o futuro potencial reprodutivo dos mesmos. A circunferência escrotal é um importante parâmetro utilizado na avaliação do potencial reprodutivo de touros adultos, por apresentar correlações com determinadas características seminais.

O valor médio para $C E$ encontrado neste trabalho $(24,8 \mathrm{~cm})$ para touros Nelore aos 18 meses de idade foi inferior ao verificado por Pinto et al. (1989) $(28,5 \mathrm{~cm})$, talvez devido à diferença do número de animais avaliados. Quando o CE é analisado em grupos de acordo com a densidade seminal, verifi- 
ca-se que os grupos de animais que possuem sêmen de baixa densidade possuem menor CE e vice-versa. Chegase ao extremo de $90 \%$ dos animais com CE abaixo de $23 \mathrm{~cm}$ possuírem sêmen oligospérmico ou azoospérmico, enquanto apenas $6,8 \%$ dos que possuem $C E \geq 27 \mathrm{~cm}$ apresentarem as mesmas características. Tal fato pode ser explicado pela alta correlação positiva entre CE e produção espermática (Toelle, 1985). Apesar de a colheita de sêmen por eletroejaculação não proporcionar sêmen com características semelhantes ao do ejaculado obtido em cobertura natural, parece ser o único método viável a campo em animais jovens, e quanto ao aspecto densidade, satisfaz às exigências quando se caracteriza o sêmen em três categorias azoospérmico, oligospérmico e denso -, independentemente deste último ser pouco ou muito denso. Verifica-se neste trabalho que o percentual de motilidade progressiva é maior em animais pertencentes ao grupo com maior CE. Observase, inversamente, que o percentual de patologia espermática é menor no grupo de animais com maior CE. Esta condição foi observada por Foote et al. (1976) e Rao Veeramachanen

\section{Referências bibliográficas}

CARDOSO, F.M. Desenvolvimento dos órgãos genitais masculinos de zebus (Bos indicus) da raça Nelore do período fetal aos 36 meses de idade. Belo Horizonte, 1977. 140 p. (Mestrado) - Inst. Ciências Biol. UFMG.

CARDOSO, F.M., GODINHO, H.P. Morphological events occurring in seminiferous tubules of the Brasilian Nelore zebu associated with puberty. Anat. Anz., v. 145, p. 262-267, 1979.

FONSECA, V. Avaliação da capacidade reprodutiva de touros Nelore: aspectos andrológicos e comportamentais. Belo Horizonte, 1995. 37 p. Monografia. Esc. Vet. UFMG.

FOOTE,R.H., SEIDEL, J.R., HAHN, J., BERNDTSON,W.E., COULTER,G.H. Seminal quality, spermatozoal outpost, and testicular changes in growing Holstein bulls: Journal of dairy Science, v. 60, n.1, p.85$88,1976$. et al. (1986). Segundo Fonseca (1995), ocorrem em fase de amadurecimento sexual alterações na espermatogênese, o que explica o alto percentual de anomalias no sêmen. Dos 100 animais estudados, 34 apresentaram sêmen com $60 \%$ ou mais de espermatozóides com motilidade progressiva, e apenas sete animais apresentaram $30 \%$ ou menos de patologia espermática. Somente em seis animais foram observadas as duas condições simultaneamente, que são consideradas mínimas para o sêmen ser classificado como de alta qualidade. Em nosso trabalho observamos que a maioria dos touros Nelore aos 18 meses de idade já havia alcançado a puberdade, estando, porém, em fase de amadurecimento sexual. Sabe-se que desde a puberdade até atingir a completa maturidade sexual ocorre uma melhora gradativa na qualidade seminal (Vale Filho, 1997). Conclui-se que a maioria dos touros Nelore aos 18 meses de idade são púberes, porém a qualidade seminal ainda é baixa. A CE pode ser considerada eficaz na seleção de touros jovens por apresentar, nesta ocasião, correlação com parâmetros estudados de qualidade seminal.

PINTO, P.A., SILVA, P.R., ALBUQUERQUE, L.G., BEZERRA, L.A.F. Avaliação da biometria testicular e capacidade de monta em bovinos das raças guzerá e Nelore. Rev. Bras. Reprod. Anim., v. 13, n. 3 , p.151-156, 1989.

RAO VEERAMACHANENI, D.N., OTT, R.S., HEATH, E.H., MCENTEE, K., BOLT, D.J., HIXON, J.E. Pathophysiology of small testes in beef bulls: relationship between scrotal circunference, histopatologic features of testes and epididymes, seminal characteristics, and endocrine profiles. American Journal of Veterinary Research. v. 47, n. 9, p.1988-1999, 1986.

TOELLE, V.D.; ROBSON, W. Estimates of genetics correlation between testicular measurements and female reproductive traints in cattle. J. Anim. Sci. v. 60, p. 89-100, 1985.

VALLE FILHO, V.R., BERGMANN, J.A.G., ANDRADE, V.J., QUIRINO, C.R., REIS, S.R., MENDONÇA, R.M.A. Caracterização andrológica de touros Nelore, selecionados para a primeira estação de monta. Rev. Bras. Reprod. Anim. v. 21, n. 2, 1997. 